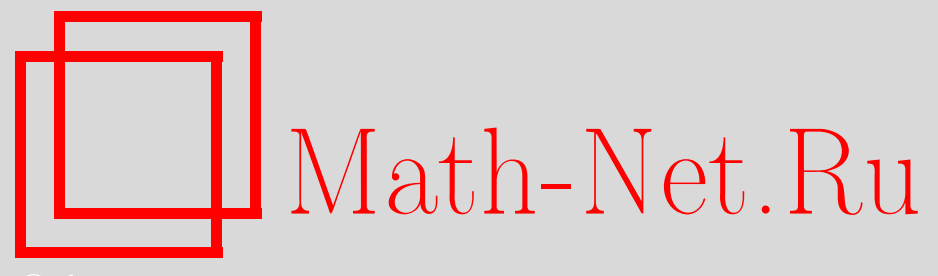

Д. Д. Лозовану, Сетевые модели дискретного оптимального управления и динамические игры с $p$ игроками, Дискрет. матем., 2001, том 13, выпуск 4, 126-143

DOI: https://doi.org/10.4213/dm306

Использование Общероссийского математического портала Math-Net.Ru подразумевает, что вы прочитали и согласны с пользовательским соглашением http://www . mathnet.ru/rus/agreement

Параметры загрузки:

IP : 35.174 .16 .151

26 апреля 2023 г., 12:01:33 


\title{
Сетевые модели дискретного оптимального управления и динамические игры с $p$ игроками
}

(C) 2001 г. Д. Д. Лозовану

\begin{abstract}
Рассматривается сетевая постановка задачи дискретного оптимального управления с закрепленными концами и формулируется ее игровой вариант с $p$ игроками. Исследуется вопрос о существовании оптимальных стратегий игроков в смысле Нэша для сетевой динамической модели игры с $p$ игроками и приводятся результаты, на базе которых предлагаются полиномиальные алгоритмы решения поставленных задач.
\end{abstract}

\section{1. Введение}

В работе рассматривается задача дискретного оптимального управления с закрепленными концами, когда динамика системы описывается с помощью ориентированного графа переходов ее состояний [1-3]. Вершины графа в этой задаче соответствуют состояниям управляемой системы, а дуги указывают на возможные ее переходы из одного состояния в другое. Дугам ориентированного графа поставлены в соответствие зависящие от времени функции, которые отражают стоимости переходов системы. Граф переходов, на дугах которого определены зависящие от времени функции стоимости дуг и в котором выделены две вершины, соответствующие начальному и конечному состояниям системы, назовем динамической сетью.

Основные результаты работы связаны с разработкой алгоритмов выбора оптимальной стратегии переходов системы из начального состояния в конечное состояние системы по дугам динамической сети. Формулируется игровой вариант этой задачи на динамической сети с $p$ игроками и исследуется вопрос о существовании оптимальных в смысле Нэша стратегий сторон. Приводятся результаты, на базе которых предлагаются эффективные полиномиальные алгоритмы решения поставленных задач. Рассматриваемые в работе задачи обобщают известную из комбинаторной оптимизации задачу о кратчайшем пути во взвешенном ориентированном графе [4], некоторые классические задачи дискретного оптимального управления из [2] и задачи о выборе оптимальных путей в игровых сетях из [5-7]. 


\section{2. Сетевая постановка задачи и метод}

\section{динамического программирования}

Пусть задана дискретная динамическая система $L$ с конечным множеством состояний $V,|V|=n$, которая в каждый момент времени $t=0,1,2, \ldots$ находится в состоянии $v(t) \in V$. В множестве $V$ выделены два состояния $v_{0}, v_{0}=v(0)$, и $v_{f}$, которые назовем соответственно начальным и конечным состояниями системы. Будем считать, что динамика системы описывается с помощью ориентированного графа переходов

$$
G=(V, E), \quad|E|=m,
$$

дуга $e=(u, v)$ которого означает возможность перехода системы $L$ из состояния $u=v(t)$ в состояние $v=v(t+1)$ для любого момента времени $t=0,1,2, \ldots$ Каждой дуге $e=(u, v)$ соответствует функция $c_{e}(t)$, которая отражает стоимость перехода системы $L$ из состояния $v(t)=u \in V$ в состояние $v(t+1)=v \in V$ в момент времени $t$.

Рассмотрим задачу поиска последовательности переходов

$$
(v(0), v(1)),(g(v(1), v(2)), \ldots,(v(T-1), v(T)) \in E,
$$

которая за период времени $[0, T]$ переводит систему $L$ из состояния $\left.v_{0}=v(0)\right)$ в состояние $v_{f}=v(T)$ и сумма стоимостей переходов которой по траектории

$$
v_{0}=v(0), v(1), v(2), \ldots, v(t)=v_{f}
$$

является минимальной. Иными словами, рассмотрим задачу, в которой требуется найти

$$
F_{v_{0} v_{f}}(T)=\min _{\left\{v_{0}=v(0), v(1), v(2), \ldots, v(T)=v_{f}\right\}} \sum_{t=0}^{T-1} c_{(v(t), v(t+1))}(t) .
$$

Заметим, что для $F_{v_{0}, v(t)}(\dot{t}), t=0,1,2, \ldots, T$, справедливо рекуррентное соотношение

$$
F_{v_{0} v(t)}(t)=\min _{v(t-1) \in V_{G}^{-}(v(t))}\left\{F_{v_{0} v(t-1)}(t-1)+c_{(v(t-1), v(t))}(t-1)\right\}
$$

где

$$
V_{G}^{-}(u)=\{v \in V \mid e=(v, u) \in E\} .
$$

Поэтому, если добавить к этим соотношениям условие

$$
F_{v_{0}, v(0)}(0)=0 \text {, }
$$

то можно табулировать значения функции $F_{v_{0}, v(t)}(t)$ при $t=0,1,2, \ldots, T$ методом динамического программирования. Таким образом, если значения функций стоимости дуг $c_{(v(t), v(t+1))}(t)$ для любого момента времени $t$ могут быть найдены непосредственно, то поставленную задачу, с заданным фиксированным $T$, можно решить за $O\left(n^{2} T\right)$ элементарных операций, использовав порядка $O(n T)$ ячеек памяти.

Нетрудно убедиться, что поставленная задача может быть решена и в случае, когда момент $T$ не фиксирован, а возможные его значения принадлежат некоторому заданному отрезку времени $\left[T_{1}, T_{2}\right]$. Решение задачи в этом случае сводится к решению $T_{2}-T_{1}+1$ задач с фиксированным $T$. Точнее, если $T \in\left[T_{1}, T_{2}\right]$, то последовательно фиксируя

$$
T=T_{1}, \quad T=T_{1}+1, \quad T=T_{1}+2, \quad \ldots, \quad T=T_{2},
$$


и каждый раз решая задачу с заданным $T$, мы сможем из всех полученных решений выбрать решение, для которого оптимальное значение функции цели является наименьшим.

В дальнейшем мы будем рассматривать случай, когда $T \in[0, n-1]$ и оптимальная траектория переходов системы в $G$ соответствует простому пути из $v_{0}$ в $v_{f}$. Одним из условий, гарантирующим выполнение такого свойства решения задачи, является положительность и неубываемость функций стоимости переходов динамической системы по дугам динамической сети. Итак, будем предполагать, что для рассматриваемой задачи нет ограничений на количество переходов системы и что оптимальная траектория в $G$ соответствует простому пути из $v_{0}$ в $v_{f},(T \leqslant n-1)$. В этом случае для нас полезно будет рассматривать задачу в терминах стратегий переходов системы на динамической сети. Стратегию переходов системы $L$ на динамической сети с начальным состоянием $v_{0}$ и конечным состоянием $v_{f}$ определим как отображение

$$
s: v \rightarrow u \in V^{+}(v), \quad v \in V \backslash\left\{v_{f}\right\}
$$

где

$$
V^{+}(v)=\{u \in V \mid e=(v, u) \in E\} .
$$

Для произвольной стратегии $s$ множество дуг вида $(v, s(v))$ порождает в $G$ подграф $G_{s}=\left(V, E_{s}\right)$, который обладает таким свойством, что либо в $G_{s}$ существует единственный ориентированный путь $P_{s}\left(v_{0}, v_{f}\right)$ из $v_{0}$ в $v_{f}$, либо такого пути в нем нет. В последнем случае при движении в $G_{s}$ по исходящим дугам из $v_{0}$ мы однозначно попадем на некоторый ориентированный цикл $C_{s}$. Если в $G_{s}$ существует ориентированный путь $P_{s}\left(v_{0}, v_{f}\right)$ из $v_{0}$ в $v_{f}$, то двигаясь по дугам этого пути из $v_{0}$ в $v_{f}$, мы сможем пронумеровать дуги числами $0,1,2, \ldots, k_{s}$. Эти числа характеризуют моменты времени $t_{e}(s)$ перехода системы из одного состояния в другое при использовании стратегии $s$.

Для заданной стратегии

$$
s: v \rightarrow u \in V^{+}(v), \quad v \in V \backslash\left\{v_{f}\right\},
$$

определим величину

$$
F_{v_{0} v_{f}}(s)=\sum_{e \in E\left(P_{s}\left(v_{0}, v_{f}\right)\right)} c_{e}(t(s)),
$$

если в $G_{s}$ существует путь $P_{s}\left(v_{0}, v_{f}\right)$ из $v_{0}$ в $v_{f}$. Если в $G_{s}$ такого пути нет, то полагаем $F_{v_{0} v_{f}}(s)=\infty$.

Таким образом, задачу дискретного оптимального управления на динамической сети можно рассматривать как задачу отыскания стратегии $s^{*}$, для которой

$$
F_{v_{0} v_{f}}\left(s^{*}\right)=\min _{s \in S} F_{v_{0} v_{f}}(s),
$$

где $S$ - множество допустимых стратегий, определяемое структурой графа $G$.

\section{3. Сетевая динамическая модель игры с $p$ игроками и задача выбора оптимальных стратегий игроков в смысле Нэша}

Пусть задана динамическая система $L$ с конечным множеством состояний $V,|V|=n$, которая в момент времени $t=0,1,2, \ldots$ находится в состоянии $v(t) \in V$. Так же, 
как и в разделе 2 , будем предполагать, что динамика системы описывается ориентированным графом переходов $G=(V, E)$, в котором выделены вершины $v_{0}$ и $v_{f}$, соответствующие начальным и конечным состояниям системы $L$.

Будем считать, что множество $V$ разбито на $p$ непересекающихся подмножеств

$$
V_{1}, V_{2}, \ldots, V_{p}, \quad V=\bigcup_{i=1}^{p} V_{i}, \quad V_{i} \cap V_{j}=\varnothing, \quad i \neq j
$$

и вершины $v \in V_{i}$ будем рассматривать как позиции игрока $i, i=1, \ldots, p$. Кроме того, предположим, что каждой дуге $e=(u, v) \in E$ поставлено в соответствие $p$ функций

$$
c_{e}^{1}(t), c_{e}^{2}(t), \ldots, c_{e}^{p}(t)
$$

где $c_{e}^{i}(t)$ представляет собой стомость перехода системы $L$ из состояния $u=u(t)$ в состояние $v=v(t+1)$ на отрезке времени $[t, t+1]$ для игрока $i, i=1,2, \ldots, p$.

Определим стратегии игроков $1,2, \ldots, p$ как отображения $s_{1}, s_{2}, \ldots, s_{p}$ на подмножествах $V_{1}, V_{2}, \ldots, V_{p}$, соответственно,

$$
\begin{aligned}
s_{1}: v \rightarrow u \in V_{G}(v), & v \in V_{1} \backslash\left\{v_{f}\right\}, \\
s_{2}: v & \rightarrow u \in V_{G}(v), \quad v \in V_{2} \backslash\left\{v_{f}\right\}, \\
& \ldots \\
s_{p}: v & \rightarrow u \in V_{G}(v), \quad v \in V_{p} \backslash\left\{v_{f}\right\},
\end{aligned}
$$

где $V_{G}(v)$ является множеством концов всех дуг, исходящих из $v$, то есть

$$
V_{G}(v)=\{u \in V \mid e=(v, u) \in E\} .
$$

Для заданного набора стратегий игроков $s=\left(s_{1}, s_{2}, \ldots, s_{p}\right)$ обозначим через $H_{s}=\left(V, E_{s}\right)$ подграф, порожденный множеством дуг вида

$$
e=\left(v, s_{i}(v)\right), \quad v \in V \backslash\left\{v_{f}\right\}, \quad i=1, \ldots, p .
$$

Очевидно, что для фиксированных стратегий $s_{1}, s_{2}, \ldots, s_{p}$ либо в $H_{s}$ существует единственный ориентированный путь $P_{s}\left(v_{0}, v_{f}\right)$ из $v_{0}$ в $v_{f}$, либо такого пути в нем нет. В последнем случае, если мы двигаемся по исходящим дугам из $v_{0}$, то однозначным образом попадем на некоторый ориентированный цикл $\boldsymbol{C}_{\boldsymbol{s}}$.

Для заданного набора стратегий игроков $s_{1}, s_{2}, \ldots, s_{p}$ и заданными начальным и конечным состояниями $v_{0}$ и $v_{f}$ определим величины

$$
F_{v_{0} v_{f}}^{1}\left(s_{1}, s_{2}, \ldots, s_{p}\right), F_{v_{0} v_{f}}^{2}\left(s_{1}, s_{2}, \ldots, s_{p}\right), \ldots, F_{v_{0} v_{f}}^{p}\left(s_{1}, s_{2}, \ldots, s_{p}\right)
$$

следующим образом. Пусть в подграфе $H_{s}$ существует путь $P_{s}\left(v_{0}, v_{f}\right)$ с множеством дуг $E\left(P_{s}\left(v_{0}, v_{f}\right)\right)$, тогда этот путь является единственным и мы сможем пронумеровать дуги числами $0,1,2, \ldots, k_{s}$ по мере движения из начальной вершины $v_{0}$ в конечную вершину $v_{f}$. Эти числа характеризуют моменты времени $t_{e}\left(s_{1}, s_{2}, \ldots, s_{p}\right)$ перехода системы $L$ по дугам $e \in E\left(P_{s}\left(v_{0}, v_{f}\right)\right)$ из одного состояния в. другое, если игроки зафиксировали свои стратегии $s_{1}, s_{2}, \ldots, s_{p}$ переходов по дугам динамической сети. В этом случае мы полагаем

$$
F_{v_{0} v_{f}}^{i}\left(s_{1}, s_{2}, \ldots, s_{p}\right)=\sum_{e \in E\left(P_{s}\left(v_{0}, v_{f}\right)\right)} c_{e}^{i}\left(t_{e}\left(s_{1}, s_{2}, \ldots, s_{p}\right)\right), \quad i=1, \ldots, p .
$$

5 Дискретная математика, т.13 $N 24$ 
Если в $H_{s}$ не существует ориентированного пути $P_{s}\left(v_{0}, v_{f}\right)$ из $v_{0}$ в $v_{f}$, то полагаем

$$
F_{v_{0} v_{f}}^{i}\left(s_{1}, s_{2}, \ldots, s_{p}\right)=\infty
$$

Рассмотрим задачу поиска стратегий $s_{1}^{*}, s_{2}^{*}, \ldots, s_{p}^{*}$, для которых

$$
F_{v_{0} v_{f}}^{i}\left(s_{1}^{*}, s_{2}^{*}, \ldots, s_{i-1}^{*}, s_{i}^{*}, s_{i+1}^{*}, s_{p}^{*}\right) \leqslant F_{v_{0} v_{f}}^{i}\left(s_{1}^{*}, s_{2}^{*}, \ldots, s_{i-1}^{*}, s_{i}, s_{i+1}^{*}, s_{p}^{*}\right)
$$

для всех

$$
s_{i} \in S_{i}, \quad S_{i}=\left\{s_{i}: v \rightarrow u \in V_{G}(v), v \in V_{i}\right\}, \quad i=1,2, \ldots, p .
$$

Таким образом, мы сформулировали задачу нахождения оптимальных стратегий в смысле Нэша $[8,9]$ для игры в нормальной форме, определенной множествами стратегий $S_{1}, S_{2}, \ldots, S_{p}$ игроков $1,2, \ldots, p$ и платежными функциями

$$
F_{v_{0} v_{f}}^{1}\left(s_{1}, s_{2}, \ldots, s_{p}\right), F_{v_{0} v_{f}}^{2}\left(s_{1}, s_{2}, \ldots, s_{p}\right), \ldots, F_{v_{0} v_{f}}^{p}\left(s_{1}, s_{2}, \ldots, s_{p}\right) .
$$

В позиционной форме эта игра определяется графом $G$, разбиением $V_{1}, V_{2}, \ldots, V_{p}$, векторами функций

$$
c^{i}(t)=\left(c_{e_{1}}^{i}(t), c_{e_{2}}^{i}(t), \ldots, c_{e_{m}}^{i}(t)\right), \quad i=1, \ldots, p,
$$

начальной и конечной позициями $v_{0}, v_{f}$. Назовем эту игру $c$-игрой $p$ игроков на динамической игровой сети

$$
\left(G, V_{1}, V_{2}, \ldots, V_{p}, c^{1}(t), c^{2}(t), \ldots, c^{p}(t), v_{0}, v_{f}\right)
$$

Если $V=V_{1}$, то мы получаем задачу дискретного оптимального управления с фиксированными концами $[2,10]$. В случае, когда функции $c_{e}^{i}(t), e \in E, i=1, \ldots, p$, являются постоянными и $V=V_{1}$, задача превращается в задачу о кратчайшем пути во взвешенном ориентированном графе [4]. Если же $V=V_{1} \cup V_{2}$ и $c^{2}=-c^{1}$, то при постоянных функциях стоимости дуг мы получаем задачу о минимаксном пути из $[4,6]$. Случай, когда

$$
V=V_{1} \cup V_{2} \cup \ldots \cup V_{p}
$$

и все функции $c_{e}^{i}(t), e \in E, i=1, \ldots, p$, являются постоянными, исследовался в [11]. Общая модель с произвольными $c_{e}^{i}(t)$ рассматривалась в [10].

Сформулированная модель может быть интерпретирована как динамическая игра $p$ игроков с интегральным по времени платежом для каждого из игроков, где $v_{0}$ и $v_{f}$ представляют собой соответственно начальную и конечную позицию игры, а $V_{1}, V_{2}, \ldots, V_{p}$ - соответствующие множества позиций игроков. Если система $L$ имеет начальное состояние $v_{0}=v(0)$ и $v_{0} \in V_{i}$, то в момент времени $t=0$ выбор перехода из состояния $v_{0}=v(0)$ принадлежит игроку $i$. В общем случае, в момент времени $t$ ход осуществляет игрок $i$, если система $L$ находится в позиции $v(t)=v \in V_{i}$. Переход из состояния $v=v(t)$ (позиции $v$ ) в состояние $u=u(t+1)$ осуществляется по дугам $(v, u) \in E$ и называется ходом игрока $i$. Игра может быть конечной или бесконечной. Если состояние $v_{f}$ достигается в момент времени $t,\left(v_{f}=v(t)\right)$, то игра заканчивается, то есть игра является конечной. Если же состояние $v_{f}$ не достигается за конечное время, то игра является бесконечной. Каждый игрок, осуществляя свои ходы в этой игре, старается минимизировать суммарные стоимости переходов по траектории. 


\section{4. Условие регулярности для динамических сетей и алгоритм решения задачи в случае $p=1$}

Сформулированная в разделе 3 модель в случае $p=1$ превращается в обычную задачу дискретного оптимального управления на сети $G$ с заданными функциями стоимости переходов $c_{e}(t), e \in E$, начальным состоянием $v_{0}$ и конечным состоянием $v_{f}$. Если функции $c_{e}(t), e \in E$, являются положительными и неубывающими, то оптимальная траектория переходов системы из $v_{0}$ в $v_{f}$ порождает в $G$ путь $P^{*}\left(v_{0}, v_{f}\right)$, который содержит не более $n-1$ дуг. Поэтому, если в $G$ вершина $v_{f}$ достижима из $v_{0}$, то оптимальный путь $P^{*}\left(v_{0}, v_{f}\right)$ из $v_{0}$ в $v_{f}$ может быть найден методом динамического программирования так, как указано в разделе 2. Ниже покажем, что для поиска оптимальных путей в динамической сети может быть использован более эффективный алгоритм, если динамическая сеть удовлетворяет следующему условию. Пусть в $G$ произвольный оптимальный путь $P^{*}\left(v_{0}, v\right)$ из $v_{0}$ в $v, v \in V$, обладает тем свойством, что если он представим в виде

$$
P^{*}\left(v_{0}, v\right)=P_{1}^{*}\left(v_{0}, w\right) \cup P_{2}^{*}(w, v)
$$

где $\left(P_{1}^{*}\left(v_{0}, w\right)\right.$ и $P_{2}^{*}(w, v)$ не имеют общих дуг, то лидирующая часть $P_{1}^{*}\left(v_{0}, w\right)$ представляет собой оптимальный путь из $v_{0}$ в $w$. Если это условие выполняется в $G$, то будем говорить, что динамическая сеть удовлетворяет условию регулярности.

Пусть динамическая сеть $G$ удовлетворяет условию регулярности, тогда следующий алгоритм находит оптимальные пути $P^{*}\left(v_{0}, v\right)$ из $v_{0}$ до $v \in V$.

Шаг 0. Полагаем $U=\left\{v_{0}\right\}, E^{*}=\varnothing$. Каждой вершине $v \in V$ ставим в соответствие величины $F(v)$ и $t(v)$, где

$$
\begin{array}{rlrl}
F\left(v_{0}\right) & =0, \quad F(v)=\infty, & v \in V \backslash\left\{v_{0}\right\} \\
t\left(v_{0}\right) & =0, \quad t(v)=\infty, \quad v \in V \backslash\left\{v_{0}\right\} .
\end{array}
$$

Шаг $k$. Находим множество

$$
\begin{aligned}
E^{\prime} & =\left\{\left(u^{\prime}, v^{\prime}\right) \in E(U) \mid F\left(u^{\prime}\right)+c_{\left(u^{\prime}, v^{\prime}\right)}\left(t\left(u^{\prime}\right)\right)\right. \\
& =\min _{u \in U} \min _{v \in \bar{V}(u)}\left\{F(u)+c_{(u, v)}(t(u))\right\},
\end{aligned}
$$

где

$$
\begin{aligned}
& E(U)=\{(u, v) \in E \mid u \in U, v \in V \backslash U\} \\
& \bar{V}(u)=\{v \in V \backslash U \mid(u, v) \in E(U)\}
\end{aligned}
$$

Выделяем множество вершин

$$
V^{\prime}=\left\{v^{\prime} \in V \backslash U \mid\left(u^{\prime}, v^{\prime}\right) \in E^{\prime}\right\} .
$$

Для каждого $v^{\prime} \in V^{\prime}$ выбираем одну дугу $\left(u^{\prime}, v^{\prime}\right) \in E^{\prime}$ и находим объединение $\bar{E}^{\prime}$ таких дуг. После этого меняем величины $F\left(v^{\prime}\right)$ и $t\left(v^{\prime}\right)$ для каждой вершины $v^{\prime} \in V^{\prime}$ следующим образом:

$$
F\left(v^{\prime}\right)=F\left(u^{\prime}\right)+c_{\left(u^{\prime}, v^{\prime}\right)}\left(t\left(u^{\prime}\right)\right), t\left(v^{\prime}\right)=t\left(u^{\prime}\right)+1, \quad\left(u^{\prime}, v^{\prime}\right) \in \bar{E}^{\prime} .
$$

Заменяем множество $U$ на $U \cup V^{\prime}$ и $E^{*}$ на $E^{*} \cup \bar{E}^{\prime}$. Полагаем $V^{k}=U, E^{k}=E^{*}$. Если $V^{k} \neq V$, то фиксируем дерево $H^{k}=\left(V^{k}, E^{k}\right)$, затем переходим к шагу $k+1$, в противном случае фиксируем дерево $H^{*}=\left(V, E^{*}\right)$ и заканчиваем работу. 
Замечание 1. В $G$ дерево $H^{*}=\left(V, E^{*}\right)$ представляет собой дерево оптимальных путей от вершины $v_{0}$ до каждой вершины $v \in V$, а дерево $H^{k}=\left(V^{k}, E^{k}\right)$ представляет собой часть дерева $H^{*}$, полученную после $k$ итераций алгоритма. Если в $G$ необходимо найти оптимальный путь от вершины $v_{0}$ до вершины $v_{f}$, то алгоритм может прекратить свою работу после $k$-й итерации, как только будет выполнено условие $v_{f} \in V^{k}$. Иными словами, в этом случае условие $V^{k} \neq V$ в алгоритме может быть заменено условием $v_{f} \notin V^{k}$. Величины $F(v), v \in V$, после работы алгоритма равны суммарной стоимости дуг оптимальных путей от $v_{0}$ до $v \in V$, a $t(v)$ равно числу дуг этих путей.

Теорема 1. Пусть в $G$ функции $c_{e}(t), e \in E$, положительнъ. Если динамическая сеть удовлетворяет условию регулярности, то дерево $H^{k}=\left(V^{k}, E^{k}\right)$, полученное после $k$ итерачий алгоритма, представляет собой дерево оптималънъх путей от вериинь $v_{0}$ до вершинъ $v \in V^{k}$ для динамической сети.

Доказательство. Доказательство теоремы проведем индукцией по числу итераций алгоритма. Очевидно, что если $k=1$, то теорема справедлива.

Предположим, что теорема справедлива для $k \leqslant r$. Покажем, что утверждение теоремы справедливо для $k=r+1$. Если $H^{r}=\left(V^{r}, E^{r}\right)-$ дерево, полученное после $r$ шагов алгоритма, а $H^{r+1}=\left(V^{r+1}, E^{r+1}\right)$ - дерево, полученное после $r+1$ шага алгоритма, то $V^{\circ}=V^{r+1} \backslash V^{r}$ и $E^{\circ}=E^{r+1} \backslash E^{r}$ представляют собой множество вершин и множество дуг, полученных непосредственно на $r+1$ шаге алгоритма. Покажем, что если $v^{\prime}$ - произвольная вершина из $V^{\circ}$ для $H^{r+1}$, то единственный в $H^{r+1}$ путь $P_{H^{r+1}}^{*}\left(v_{0}, v^{\prime}\right)$ из $v_{0}$ в $v^{\prime}$ является оптимальным в динамической сети $\left(G, c(t), v_{0}, v_{f}\right)$. Действительно, если это не так, то существует оптимальный путь $Q\left(v_{0}, v^{\prime}\right)$ из $v_{0}$ в $v^{\prime}$, который не содержит дугу $e=\left(w^{\prime}, v^{\prime}\right) \in E^{\circ}$. Путь $Q\left(v_{0}, v^{\prime}\right)$ может быть представлен в виде

$$
Q\left(v_{0}, v^{\prime}\right)=Q^{1}\left(v_{0}, u^{\prime}\right) \cup\left\{\left(u^{\prime}, v\right)\right\} \cup Q^{2}\left(v, v^{\prime}\right)
$$

где $u^{\prime}$ является последней вершиной, принадлежащей множеству $V^{r}$ при движении от $v_{0} \mathrm{\kappa} v^{\prime}$ по дугам $Q\left(v_{0}, v^{\prime}\right)$. Покажем, что

$$
\operatorname{cost}\left(Q\left(v_{0}, v^{\prime}\right)\right)>\operatorname{cost}\left(P^{*}\left(v_{0}, v^{\prime}\right)\right)
$$

где

$$
\operatorname{cost}\left(Q\left(v_{0}, v^{\prime}\right)\right)=\sum_{t=0}^{m_{Q}} c_{e_{t}}(t)
$$

$e_{0}, e_{1}, \ldots, e_{m_{Q}}$ представляют собой дуги пути $Q\left(v_{0}, v^{\prime}\right)$, когда движение осуществляется от $v_{0} \mathrm{~K} v^{\prime}$,

$$
\operatorname{cost}\left(P^{*}\left(v_{0}, v^{\prime}\right)\right)=\sum_{t=0}^{m_{p}} c_{e_{t}^{\prime}}(t),
$$

$e_{0}^{\prime}, e_{1}^{\prime}, \ldots, e_{m_{p}}^{\prime}$ представляют собой дуги пути $P^{*}\left(v_{0}, v^{\prime}\right)$, когда движение осуществляется от $v_{0} \mathrm{~K} v^{\prime}$.

Поскольку условие регулярности на динамической сети $\left(G, c(t), v_{0}, v_{f}\right)$ выполняется, согласно предположению индукции

$$
F\left(u^{\prime}\right)=\operatorname{cost}\left(Q\left(v_{0}, u^{\prime}\right)\right)
$$


Кроме того, согласно описанию алгоритма

$$
F\left(u^{\prime}\right)+c_{\left(u^{\prime}, v^{\prime}\right)}\left(t\left(u^{\prime}\right)\right)>F\left(w^{\prime}\right)+c_{\left(w^{\prime}, v^{\prime}\right)}\left(t\left(w^{\prime}\right)\right)=F\left(v^{\prime}\right),
$$

где $e^{\prime}=\left(w^{\prime}, v^{\prime}\right)$ - последняя дуга пути $P^{*}\left(v_{0}, v^{\prime}\right)$. Тогда

$$
\operatorname{cost}\left(Q^{1}\left(v_{0}, u^{\prime}\right) \cup\left\{\left(u^{\prime}, v\right)\right\}\right)>\operatorname{cost}\left(P^{*}\left(v_{0}, v^{\prime}\right)\right),
$$

поскольку

$$
F\left(u^{\prime}\right)+c_{\left(u^{\prime}, v^{\prime}\right)}\left(t\left(u^{\prime}\right)\right)=\operatorname{cost}\left(Q^{1}\left(v_{0}, u^{\prime}\right) \cup\left\{\left(u^{\prime}, v\right)\right\}\right)
$$

и $F\left(v^{\prime}\right)=\operatorname{cost}\left(P^{*}\left(v_{0}, v^{\prime}\right)\right)$.

Функции $c_{e}(t), e \in E$, являются положительными, поэтому

$$
\begin{aligned}
\operatorname{cost}\left(Q\left(v_{0}, v^{\prime}\right)\right) & =\operatorname{cost}\left(Q^{1}\left(v_{0}, u^{\prime}\right) \cup\left\{\left(u^{\prime}, v\right)\right\} \cup Q^{2}\left(v, v^{\prime}\right)\right) \\
& \geqslant \operatorname{cost}\left(Q^{1}\left(v_{0}, v^{\prime}\right) \cup\left\{\left(u^{\prime}, v\right)\right\}\right)>\operatorname{cost}\left(P^{*}\left(v_{0}, v^{\prime}\right)\right) .
\end{aligned}
$$

Это противоречит предположению, что путь $\left(Q\left(v_{0}, v^{\prime}\right)\right.$ не является оптимальным. Следовательно, дерево $H^{r+1}=\left(V^{r+1}, E^{r+1}\right)$ находит оптимальные пути $P^{*}\left(v_{0}, v\right)$ из $v_{0}$ в $v$ для всех $v \in V^{r+1}$. Теорема доказана.

Предложенный алгоритм нахождения оптимальных путей в динамической сети является расширением алгоритма Дейкстры [4]. Далее мы покажем, что подобный алгоритм может быть использован для нахождения оптимальных путей в динамических сетях с $p$ игроками, если условие регулярности в динамической сети удовлетворяется по отношению к каждому игроку $i, i=1, \ldots, p$. Условие регулярности для динамической сети с $p$ игроками

$$
\left(G, V_{1}, V_{2}, \ldots, V_{p}, c^{1}(t), c^{2}(t), \ldots, c^{p}(t), v_{0}, v_{f}\right)
$$

по отношению к игроку $i$ мы определим следующим образом.

Пусть

$$
s_{1}, s_{2}, \ldots, s_{i-1}, s_{i+1}, \ldots, s_{p}
$$

- некоторый набор стратегий для игроков $1,2, \ldots, i-1, i+1, \ldots, p$. Обозначим через $G_{S \backslash s_{i}}=\left(V, E_{S \backslash s_{i}}\right)$ подграф графа $G$, где

$$
\begin{aligned}
E_{S \backslash s_{i}} & =E_{s_{1}} \cup E_{s_{2}} \cup \cdots \cup E_{s_{i}-1} \cup E^{i} \cup E_{s_{i}+1} \cup \cdots \cup E_{p}, \\
E^{i} & =\left\{(u, v) \in E \mid u \in V_{i}\right\}, \\
E_{s_{k}} & =\left\{(u, v) \in E \mid u \in V_{k}, v=s_{k}(u)\right\}, \quad k=1 \ldots, p, \quad k \neq i .
\end{aligned}
$$

На $G_{S \backslash s_{i}}$ рассмотрим задачу дискретного оптимального управления с заданными функциями стоимости переходов $c_{e}^{i}(t)$ для $e \in E_{S \backslash s_{i}}$, начальным состоянием $v_{0}$ и конечным состоянием $v$. Если для любого набора стратегий $s_{1}, s_{2}, \ldots, s_{i-1}, s_{i+1}, \ldots, s_{p}$ функции стоимости $c_{e}^{i}(t)$ в $G_{S \backslash s_{i}}$ обладают свойством, что любой оптимальный путь $P^{*}\left(v_{0}, w\right)$, который представим в виде

$$
P^{*}\left(v_{0}, w\right)=P_{1}^{*}\left(v_{0}, w\right) \cup P_{2}^{*}(w, v),
$$

где $P_{1}^{*}\left(v_{0}, w\right)$ и $P_{2}^{*}(w, v)$ не имеют общих дуг, лидирующая его часть $P_{1}^{*}\left(v_{0}, w\right)$ представляет собой оптимальный путь из $v_{0}$ в $w$, то будем говорить, что для динамической сети

$$
\left(G, V_{1}, V_{2}, \ldots, V_{p}, c^{1}(t), c^{2}(t), \ldots, c^{p}(t), v_{0}, v_{f}\right)
$$


с $p$ игроками выполняется условие регулярности относительно игрока $i$.

Нетрудно убедиться, что если функции

$$
c_{e}^{i}(t), \quad i=1, \ldots, p, \quad e \in E,
$$

являются постоянными положительными функциями, то условие регулярности для динамической сети с $p$ игроками выполняется относительно каждого игрока $i, i=$ $1, \ldots, p$. Легко также проверить, что условие регулярности соблюдается относительно каждого игрока для динамической сети, если

$$
c_{e}^{i}(t)=f^{i}(t), \quad i=1, \ldots, p, \quad e \in E,
$$

где $f^{1}(t), f^{2}(t), \ldots, f^{p}(t)$ - произвольный набор положительных и неубывающих функций. Если динамическая сеть имеет структуру. графа без ориентированных циклов, то $f^{1}(t), f^{2}(t), \ldots, f^{p}(t)$ в $(3)$ могут быть произвольными неубывающими функциями. Если же $G$ имеет структуру $k$-дольного графа без ориентированных циклов, то функции стоимости переходов могут быть произвольными положительными функциями.

Нетрудно проверить, что число элементарных операций, необходимых для реализации алгоритма, предложенного в данном разделе, есть $O\left(n^{2}\right)$ (без учета вычислений значении функции $c_{e}(t)$ при заданном $\left.t\right)$, а число используемых ячеек памяти есть $O(m)$. Заметим также, что проверка выполнения условия регулярности произвольной динамической сети может быть осуществлена за $O\left(n^{3}\right)$ операций следующим образом: находим дерево $H^{*}=\left(V, E^{*}\right)$ согласно предложенному алгоритму и определяем значения $F(v)$ и $t(v)$ для каждой вершины $v \in V$, затем в найденном дереве $H^{*}$ проверяем с помощью метода динамического программирования, соответствует ли путь $P_{H^{*}}\left(v_{0}, v\right)$ от $v_{0}$ до $v$ в дереве $H^{*}$ оптимальной траектории с $T=t(v)$.

\section{5. Основные результаты}

Основные результаты работы связаны с рассмотрением вопроса существования оптимальных по Нэшу стратегий игроков в динамической $c$-игре и исследованием свойств решений задачи.

Теорема 2. Пусть

$$
\left(G, V_{1}, V_{2}, \ldots, V_{p}, c^{1}, c^{2}, \ldots, c^{p}, v_{0}, v_{f}\right)
$$

- динамическая сеть с р игроками, для которой в $G$ вершина $v_{f}$ достижима из вершинь $v_{0}$ u вектор-функция

$$
c^{i}(t)=\left(c_{e_{1}}^{i}(t), c_{e_{2}}^{i}(t), \ldots, c_{e_{m}}^{i}(t)\right)
$$

содержит положительные и неубывающие компоненты. Кроме того, пусть условие регулярности в динамической сети выполняется по отношении к каждому игроку. Тогда в динамической с-игре на сети

$$
\left(G, V_{1}, V_{2}, \ldots, V_{p}, c^{1}, c^{2}, \ldots, c^{p}, v_{0}, v_{f}\right)
$$

существуют оптималъные по Нэиу стратегии $s_{1}^{*}, s_{2}^{*}, \ldots, s_{p}^{*}$ игроков $1,2, \ldots, p$. 
Доказательство. Доказательство проведем методом математической индукции по числу игроков $p$ в динамической $c$-игре. Задача нахождения оптимальной стратегии в случае $p=1$ превращается в обычную задачу дискретного оптимального управления на $G$. Как было показано в разделе 1 , оптимальный путь $P^{*}\left(v_{0}, v_{f}\right)$ из $v_{0}$ в $v_{f}$ при положительных и неубывающих функциях стоимости переходов по дугам сети содержит не более $n-1$ дуг. Поэтому этот путь может быть найден за конечное число шагов с помощью метода динамического программирования. Если оптимальный путь $P^{*}\left(v_{0}, v_{f}\right)$ найден, то оптимальная стратегия $s_{1}^{*}$ может быть найдена следующим образом:

$$
s_{1}^{*}(u)= \begin{cases}v, & v \in V_{G}(u), v \text { произвольна, если } u \notin P^{*}\left(v_{0}, v_{f}\right), \\ v, & v \in V_{G}(u), v \text { произвольна, если } u=v_{f}, \\ v, & (u, v) \in E\left(P^{*}(u, v)\right), \text { если } u \in P^{*}\left(u, v_{f}\right), u \neq v_{f} .\end{cases}
$$

Таким образом, при $p=1$ существует оптимальная стратегия $s_{1}^{*}$.

Предположим, что теорема верна для любого $p \leqslant k, k \geqslant 1$, и покажем, что тогда она справедлива и для $p=k+1$. Пусть имеется динамическая сеть с $p=k+1$ игроками. Будем рассматривать задачу нахождения оптимальных стратегий игроков в смысле Нэша для игроков $2,3, \ldots, p$, фиксируя возможные допустимые стратегии $s_{1}^{1}, s_{1}^{2}, \ldots, s_{1}^{q}$ для первого игрока. Заметим, что если первый игрок фиксирует свою первую стратегию $s_{1}=s_{1}^{1}$ и если мы рассмотрим задачу нахождения оптимальных стратегий по Нэшу для остальных игроков $2,3, \ldots, p$, то в позиционной форме полученная игра будет представлять собой динамическую $c$-игру с $p-1$ игроками, поскольку позиции первого игрока могут считаться позициями любого другого игрока; для определенности эти позиции будем считать позициями второго игрока.

Таким образом, при $s_{1}=s_{1}^{1}$ мы получаем новую динамическую $c$-игру с $p-1$ игроками на динамической сети

$$
\left(G^{1}, V_{2}^{1}, V_{3}, \ldots, V_{p}, c^{12}, c^{13}, \ldots, c^{1 p}, v_{0}, v_{f}\right),
$$

где $V_{2}^{1}=V_{1} \cup V_{2}$, а $G^{1}=\left(V, E^{1}\right)$ является графом, полученным из $G$ удалением дуг $e=(u, v) \in E$, для которых $u \in V_{1}$ и $v \neq s_{1}^{1}(u)$. При этом $c^{1 i}(t)$ являются векторфункциями стоимостей дуг, полученными в результате сужения множества $E$ на $E^{1}$, то есть

$$
c^{1 i}(t)=\left(c_{e_{1}}^{i}(t), c_{e_{2}}^{i}(t) \ldots, c_{e_{m_{1}}}^{i}(t)\right), \quad e_{i} \in E^{1}, \quad m_{1}=\left|E^{1}\right|, \quad i=2, \ldots, p .
$$

Если мы рассмотрим игру с $p-1$ игроками в нормальной форме, то она определяется $p-1$ платежными функциями

$$
F_{v_{0} v_{f}}^{2}\left(s_{1}^{1}, s_{2}, s_{3}, \ldots, s_{p}\right), \quad F_{v_{0} v_{f}}^{3}\left(s_{1}^{1}, s_{2}, s_{3}, \ldots, s_{p}\right), \ldots, \quad F_{v_{0} v_{f}}^{p}\left(s_{1}^{1}, s_{2}, s_{3}, \ldots, s_{p}\right)
$$

где

$$
s_{2} \in S_{2}, s_{3} \in S_{3}, \ldots, s_{p} \in S_{p}
$$

Если в $G^{1}$ вершина $v_{f}$ не является достижимой из $v_{0}$, то

$$
F_{v_{0} v_{f}}^{i}\left(s_{1}^{1}, s_{2}, s_{3}, \ldots, s_{p}\right)=\infty, \quad\left(s_{2}, s_{3}, \ldots, s_{p}\right) \in S_{2} \times S_{3} \times \cdots \times S_{p}, \quad i=2, \ldots, p .
$$

Если в $G^{1}$ вершина $v_{f}$ достижима из $v_{0}$, то согласно предположению индукции для динамической $c$-игры с $p-1$ игроками существуют оптимальные стратегии игроков $s_{2}^{1^{*}}, s_{3}^{1^{*}}, \ldots, s_{p}^{1^{*}}$. 
Аналогичным образом мы можем рассмотреть случай, когда первый игрок фиксируе свою вторую стратегию $s_{1}=s_{1}^{2}$. Если в полученном графе $G^{2}$ вершина $v_{f}$ не является достижимой из $v_{0}$, то

$$
F_{v_{0} v_{f}}^{i}\left(s_{1}^{2}, s_{2}, s_{3}, \ldots, s_{p}\right)=\infty, \quad\left(s_{2}, s_{3}, \ldots, s_{p}\right) \in S_{2} \times S_{3} \times \cdots \times S_{p}, \quad i=1, \ldots, p .
$$

Если же в $G^{2}$ вершина $v_{f}$ достижима из $v_{0}$, то для полученной динамической $c$-игры с $p-1$ игроками на $G^{2}$ существуют оптимальные стратегии игроков $s_{2}^{2^{*}}, s_{3}^{2^{*}}, \ldots, s_{p}^{2^{*}}$.

Далее рассмотрим случай, когда первый игрок фиксирует свою третью стратегию $s_{1}^{3}$, тем самым найдем оптимальные стратегии $s_{2}^{3^{*}}, s_{3}^{3^{*}}, \ldots, s_{p}^{3^{*}}$ для игроков $2, \ldots, p$. Продолжая процесс, мы находим множество стратегий игроков $1,2, \ldots, p$

$$
\begin{gathered}
\left(s_{1}^{1}, s_{2}^{1^{*}}, s_{3}^{1^{*}}, \ldots, s_{p}^{1^{*}}\right), \\
\left(s_{1}^{2}, s_{2}^{2^{*}}, s_{3}^{2^{*}}, \ldots, s_{p}^{2^{*}}\right), \\
\ldots \\
\left(s_{1}^{q}, s_{2}^{q^{*}}, s_{3}^{q^{*}}, \ldots, s_{p}^{q^{*}}\right) .
\end{gathered}
$$

Среди множества этих стратегий мы выбираем множество стратегий

$$
\left(s_{1}^{j^{*}}, s_{2}^{j^{*}}, s_{3}^{j^{*}}, \ldots, s_{p}^{j^{*}}\right)
$$

для которых

$$
F_{v_{0} v_{f}}^{1}\left(s_{1}^{j^{*}}, s_{2}^{j^{*}}, \ldots, s_{p}^{j^{*}}\right)=\min _{1 \leqslant i \leqslant q} F_{v_{0} v_{f}}^{1}\left(s_{1}^{i}, s_{2}^{i^{*}}, \ldots, s_{p}^{i^{*}}\right)
$$

Тогда

$$
F_{v_{0} v_{f}}^{i}\left(s_{1}^{j^{*}}, s_{2}^{j^{*}}, \ldots, s_{i-1}^{j^{*}}, s_{i}^{j^{*}}, s_{i+1}^{j^{*}}, \ldots, s_{p}^{j^{*}}\right) \leqslant F_{v_{0} v_{f}}^{i}\left(s_{1}^{j^{*}}, s_{2}^{j^{*}}, \ldots, s_{i-1}^{j^{*}}, s_{i}, s_{i+1}^{j^{*}}, \ldots, s_{p}^{j^{*}}\right),
$$

для всех $s_{i} \in S_{i}, 2 \leqslant i \leqslant p$.

Покажем, что если условие регулярности на динамической игровой сети выполняется, то

$$
F_{v_{0} v_{f}}^{1}\left(s_{1}^{j^{*}}, s_{2}^{j^{*}}, \ldots, s_{p}^{j^{*}}\right) \leqslant F_{v_{0} v_{f}}^{1}\left(s_{1}, s_{2}^{j^{*}}, \ldots, s_{p}^{j^{*}}\right),
$$

для всех $s_{1} \in S_{1}, S_{1}=\left\{s_{1}^{1}, s_{1}^{2}, \ldots, s_{1}^{q}\right\}$.

Обозначим через $G_{s^{*}}^{\circ}=\left(V, E_{s^{*}}^{\circ}\right)$ подграф графа $G$, где

$$
\begin{aligned}
E_{s^{*}}^{\circ} & =E^{1} \cup E_{s^{*}}^{2} \cup E_{s^{*}}^{3} \cup \ldots \cup E_{s^{*}}^{p}, \\
E^{1} & =\left\{(u, v) \in E \mid u \in V_{1}\right\}, \\
E_{s^{*}}^{i} & =\left\{(u, v) \in E \mid u \in V_{i}, \quad v=s_{i}^{j^{*}}(v)\right\} .
\end{aligned}
$$

На $G_{s^{*}}^{\circ}$ рассмотрим задачу дискретного оптимального управления с функциями стоимости на дугах $c_{e}^{1}(t)$ для всех $e \in E_{s^{*}}^{\circ}$. Для нахождения оптимального пути $P_{1}^{*}\left(v_{0}, v_{f}\right)$ из $v_{0}$ в $v_{f}$, соответствующего оптимальной траектории задачи, мы сможем использовать предложенный в разделе 4 алгоритм, поскольку условие регулярности по отношению к первому игроку выюлняется на динамической игровой сети. Этот алгоритм, как легко заметить, находит путь из $v_{0}$ в $v_{f}$, порожденный стратегиями $s_{1}^{j^{*}}, s_{2}^{j^{*}}, \ldots, s_{p}^{j^{*}}$, поскольку $s_{1}^{j^{*}}, s_{2}^{j^{*}}, \ldots, s_{p}^{j^{*}}$ были выбраны согласно (4). Таким образом,

$$
F_{v_{0} v_{f}}^{1}\left(s_{1}^{j^{*}}, s_{2}^{j^{*}}, \ldots, s_{p}^{j^{*}}\right) \leqslant F_{v_{0} v_{j}}^{1}\left(s_{1}, s_{2}^{j^{*}}, \ldots, s_{p}^{j^{*}}\right)
$$

для всех $s_{1} \in S_{1}$. Теорема доказана. 
Замечание 2. Теорема 2 справедлива и в случае, когда условие регулярности на динамической сети выполняется для всех игроков за исключением лишь одного из них. Это имеет место, поскольку в доказательстве теоремы условие регулярности было использовано только на общем шаге метода математической индукции.

Замечание 3. Если $G$ не содержит ориентированных циклов, то в теореме 2 условия, что функции $c_{e}^{i}(t), i=1, \ldots, p, e \in E$, являются неубывающими, могут быть отброшены, то есть теорема 2 имеет место для произвольных положительных функций стоимости дуг динамической сети, в которой условие регулярности выполняется.

Заметим также, что если функции стоимости дуг $c_{e}^{i}(t)$ для всех $e \in E, i=1, \ldots, p$, являются положительными постоянными функциями, то оптимальные стратегии $s_{1}^{*}, s_{2}^{*}, \ldots, s_{p}^{*}$ игроков $1,2, \ldots, p$ в динамической $c$-игре не зависят от начального состояния $v_{0}$ (см. [11]). В общем случае, если $c_{e}^{i}(t)$, для всех $e \in E, i=1, \ldots, p$, зависят от времени, то оптимальные стратегии $s_{1}^{*}, s_{2}^{*}, \ldots, s_{p}^{*}$ зависят от состояния $v_{0}$.

Теорема 3. Пусть динамическая игровая сеть

$$
\left(G, V_{1}, V_{2}, \ldots, V_{p}, c^{1}, c^{2}, \ldots c^{p}, v_{0}, v_{f}\right)
$$

обладает тем свойством, что в $G$ любая вериина $v \in V$ является достижимой из вершины $v_{0}$ и вектор-функции

$$
c^{i}(t)=\left(c_{e_{1}}^{i}(t), c_{e_{2}}^{i}(t), \ldots, c_{e_{m}}^{i}(t)\right), \quad i=1, \ldots, p
$$

содержат положителъные и неубывающие компоненты. Кроме того, пусть условие регулярности для динамической игровой сети выполняется относительно каждого игрока. Тогда в $G$ существует дерево $H^{*}=\left(V, E^{*}\right)$, в котором каждая вершина $v \in V$ достижима из $v_{0} u$ единственный в $H$ ориентированный путь $P_{H^{*}}\left(v_{0}, v_{f}\right)$ из $v_{0}$ в $v_{f}$ определяет оптимальные стратегии игроков $s_{1}^{*}, s_{2}^{*}, \ldots, s_{p}^{*}$ в динамической игровой сети

$$
\left(G, V_{1}, V_{2}, \ldots, V_{p}, c^{1}, c^{2}, \ldots, c^{p}, v_{0}, v_{f}\right)
$$

где для $i=1, \ldots, p$

$$
s_{i}^{*}(u)= \begin{cases}v, & v \in V_{G}(u), v \text { произвольна, если } u \notin P_{H^{*}}\left(v_{0}, v_{f}\right), u \in V_{i}, \\ v, & v \in V_{G}(u), v \text { произволъна, если } u=v_{f}, u \in V_{i}, \\ v, & (u, v) \in P_{H^{*}}\left(v_{0}, v_{f}\right), \text { если } u \in P_{H^{*}}\left(v_{0}, v_{f}\right), u \neq v_{f}, u \in V_{i} .\end{cases}
$$

Для произволъной вершинь $w \in V \backslash\left\{v_{0}\right\}$ в дереве $H^{*}$ путь $P_{H^{*}}\left(v_{0}, w\right)$ из $v_{0}$ в $w$ определяет оптимальние стратегии $s_{1}^{*}, s_{2}^{*}, \ldots, s_{p}^{*}$ в динамической с-игре с начальной позицией игры $v_{0} u$ конечной позицией игры $w$, где для $i=1, \ldots, p$

$$
s_{i}^{*}(u)= \begin{cases}v, & v \in V_{G}(u), v \text { произволъна, если } u \notin P_{H^{*}}\left(v_{0}, w\right), u \in V_{i}, \\ v, & v \in V_{G}(u), v \text { произволъна, если } u=w, u \in V_{i}, \\ v, & (u, v) \in P_{H^{*}}\left(v_{0}, v_{f}\right), \text { если } u \in P_{H^{*}}\left(v_{0}, w\right), u \neq w, u \in V_{i} .\end{cases}
$$

Для доказательства теоремы понадобится следующая лемма. 
Лемма 1. Пусть $s_{1}^{*}, s_{2}^{*}, \ldots, s_{p}^{*}$ явяются оптимальными стратегиями игроков в динамической с-игре с динамической игровой сетъю, удовлетворяющей условиям теоремы 3. Пусть $P_{H *}\left(v_{0}, v_{f}\right)$ - ориентированный путь из $v_{0}$ в $v_{f}$ в подграфе $H_{s^{*}}=\left(V, E_{s^{*}}\right)$ графа $G$, порожсденного дугами

$$
\left(v, s_{i}^{*}(v)\right), \quad v \in V_{i} \backslash\left\{v_{f}\right\}, \quad i=1, \ldots, p .
$$

Тогда для любой вершины $w \in E\left(P_{s^{*}}\left(v_{0}, v_{f}\right)\right)$ стратегии $s_{1}^{*}, s_{2}^{*}, \ldots, s_{p}^{*}$ являются оптимальными стратегиями игроков в динамической с-игре с начальной позицией игры $v_{0} u$ конечной позицией игры $w$, то есть единственный в орграфе $H_{s^{*}}$ ориентированный путь $P_{s^{*}}^{\prime}\left(v_{0}, w\right)$ из $v_{0}$ в $w$ представляет собой часть пути $P_{s^{*}}\left(v_{0}, v_{f}\right)$ и также является оптимальным.

Доказательство. Поскольку стратегии $s_{1}^{*}, s_{2}^{*}, \ldots, s_{p}^{*}$ являются оптимальными для игроков $1,2, \ldots, p$ в динамической $c$-игре, условие (2) имеет место. Это условие означает, что при заданных

$$
s_{1}^{*}, s_{2}^{*}, \ldots, s_{i-1}^{*}, s_{i+1}^{*}, \ldots, s_{p}^{*}
$$

путь $P_{s^{*}}^{\prime}\left(v_{0}, w\right)$ является оптимальным для задачи дискретного оптимального управления (по отношении к игроку $i$ ) на графе $G^{0}=\left(V, E^{0}\right)$, порожденном дугами

$$
\left(v, s_{j}^{*}(v)\right), \quad v \in V \backslash V_{i}, \quad j=1, \ldots, p, \quad j \neq i
$$

и дугами

$$
(u, v) \in E^{i}, \quad E^{i}=\left\{(u, v) \in E \mid u \in V_{i}\right\} .
$$

Условие регулярности по отношению к игроку $i$ выполняетя на $G_{s^{*}}^{0}$, поэтому для всех $s_{i}, i=1, \ldots, p$,

$$
F_{v_{0} w}^{i}\left(s_{1}^{*}, s_{2}^{*}, \ldots, s_{i-1}^{*}, s_{i}^{*}, s_{i+1}^{*}, \ldots, s_{p}^{*}\right) \leqslant F_{v_{0} w}^{i}\left(s_{1}^{*}, s_{2}^{*}, \ldots, s_{i-1}^{*}, s_{i}, s_{i+1}^{*}, \ldots, s_{p}^{*}\right) .
$$

Лемма доказана.

Доказательство теоремъ 3. Поскольку $c_{e}^{i}(t)$, для всех $e \in E, i=1, \ldots, p$, являются положительными и неубывающими функциями и условие регулярности для динамической игровой сети выполняется по отношению к каждому игроку, на основании теоремы 2 для произвольной вершины $v \in V$ существует оптимальный путь $P_{s^{*}}\left(v_{0}, v\right)$ из $v_{0}$ в $v$, который соответствует оптимальным стратегиям $s_{1}^{*}, s_{2}^{*}, \ldots, s_{p}^{*}$ игроков для динамической $c$-игры с начальной позицией $v_{0}$ и конечной позицией $v$. Выбираем вершины $v \in V$, для которых оптимальные пути $P_{s^{*}}\left(v_{0}, v\right)$ содержат не более одной дуги. Очевидно, что граф

$$
T^{1}=\left(U^{1}, E^{1}\right),
$$

порожденный дугами таких путей имеет структуру ориентированного дерева с корнем $v_{0}$. Если $U^{1}=V$, то теорема доказана. Если же $U^{1} \neq V$, то в $V \backslash V^{1}$ существуют вершины $v \in V \backslash V^{1}$, для которых оптимальные пути $P_{s^{*}}\left(v_{0}, v\right)$ содержат две дуги. Согласно лемме 1 лидирущая часть $P_{s^{*}}^{1}\left(v_{0}, u\right)$ этих путей также является оптимальным путем из $v_{0}$ в $u$, и следовательно, все пути $P_{s^{*}}\left(v_{0}, v\right)$ с двумя дугами содержат дуги из $T^{1}$. таким образом, если к $T^{1}$ добавим последние дуги оптимальных путей $P_{s^{*}}\left(v_{0}, v\right)$, то мы получим новое дерево

$$
T^{2}=\left(U^{2}, E^{2}\right)
$$


с корнем $v_{0}$. В этом дереве произвольный путь $P_{T^{2}}\left(v_{0}, v\right)$ из $v_{0}$ в $v \in U_{2}$ явяется оптимальным. Если $U^{2}=V$, то теорема доказана. Если $U^{2} \neq V$, то находим вершины $V \backslash U^{2}$, для которых оптимальные пути $P_{s^{*}}\left(v_{0}, v\right)$ из $v_{0}$ в $v$ содержат три дуги. Присоединив концевые дуги и вершины $v$ этих путей к дереву $T^{2}$, получим дерево

$$
T^{3}=\left(U^{3}, E^{3}\right)
$$

с корневой вершиной $v_{0}$. В этом дереве любой путь $P_{T^{3}}\left(v_{0}, v\right)$ из $v_{0}$ в $v \in V^{3}$ является оптимальным. Если же $U^{3}=V$, то теорема доказана, если нет, то находим дерево

$$
T^{4}=\left(U^{4}, E^{4}\right),
$$

и так далее. Через конечное число шагов мы найдем дерево

$$
T^{q}=\left(U^{q}, E^{q}\right)
$$

для которого $U^{q}=V$ и каждый единственный в $T^{q}$ путь $P_{T^{q}}\left(v_{0}, v\right)$ из $v_{0}$ в $v$ является оптимальным, то есть $T^{q}=T^{*}$. Теорема доказана.

Далее дерево $T^{*}=\left(V, E^{*}\right)$ назовем деревом оптимальных путей для динамической $c$-игры.

Теорема 4. Пусть динамическая игровая сеть обладает тем свойством, что в $G$ любая вершина $v \in V$ является достижимой из $v_{0}$ и функции $c_{e}^{i}(t), e \in E$, $i=1, \ldots, p$, являттся положительными и неубывающими. Кроме того, пусть в динамической игровой сети выполняется условие регулярности по отношению $к$ каждому игроку. Тогда существуют $p+1$ положительные функции на $V$

$$
F^{1}: V \rightarrow \mathbf{R}^{1}, \quad F^{2}: V \rightarrow \mathbf{R}^{1}, \quad \ldots, \quad F^{p}: V \rightarrow \mathbf{R}^{1}, \quad t: V \rightarrow\{0,1,2, \ldots, n-1\}
$$

которъе удовлетворяют условиям

(1) $F^{i}\left(v_{0}\right)=0, \quad i=1, \ldots, p, \quad t\left(v_{0}\right)=0$,

(2) для любого подмножества $V^{0}$ множества $V, V^{0} \subset V$, содержащего вершину $v_{0}$, по крайней мере одно из следующих множеств $E^{1}, E^{2}, \ldots, E^{p}$, не является пустым, где при $i=1, \ldots, p$

$$
\begin{aligned}
E^{i} & =\left\{(\bar{u}, \bar{v}) \in E\left(V^{\circ}\right) \mid F^{i}(\bar{u})+c_{(\bar{u}, \bar{v})}^{i}(t(\bar{u}))\right. \\
& \left.=\min _{u \in V^{\circ} \cap V_{i}} \min _{v \in V^{\circ}(u)}\left\{F^{i}(u)+c_{(u, v)}^{i}(t(u))\right\}\right\}, \\
E\left(V^{\circ}\right) & =\left\{(u, v) \in E \mid u \in V^{\circ}, v \in V \backslash V^{\circ}\right\}, \\
V^{\circ}(u) & =\left\{v \in V \backslash V^{\circ} \mid(u, v) \in E\left(V^{\circ}\right)\right\},
\end{aligned}
$$

(3) существует вериина $v^{\prime} \in V \backslash V^{\circ}$ и дуга $\left(u^{\prime}, v^{\prime}\right) \in E^{\circ}, E^{\circ}=E^{1} \cup \ldots \cup E^{p}$, для которых

$$
F^{i}\left(u^{\prime}\right)+c_{\left(u^{\prime}, v^{\prime}\right)}^{i}\left(t\left(u^{\prime}\right)\right) \leqslant F^{i}(u)+c_{\left(u, v^{\prime}\right)}^{i}(t(u))
$$

для всех $\left(u, v^{\prime}\right) \in E^{\circ}, i=1, \ldots, p, u$

$$
F^{i}\left(v^{\prime}\right)=F^{i}\left(u^{\prime}\right)+c_{\left(u^{\prime}, v^{\prime}\right)}^{i}\left(t\left(u^{\prime}\right)\right), \quad t\left(v^{\prime}\right)=t\left(u^{\prime}\right)+1 .
$$


Для заданной вериинь $v \in V$ значения $F^{i}(v), i=1, \ldots, p$, равнь соответствующим значениям платежных функций игроков при оптималъных стратегиях $s_{1}^{*}, s_{2}^{*}, \ldots, s_{p}^{*}$, для динамической иеръ с началъной позичией $v_{0}$ и конечной позицией $v$, mo ecms

$$
F^{i}(v)=F_{v_{0}, v}^{i}\left(s_{1}^{*}, s_{2}^{*}, \ldots, s_{p}^{*}\right), \quad i=1, \ldots, p .
$$

Дерево $T^{*}=\left(V, E^{*}\right)$ оптимальных путей для динамической с-игры в $G$ находитсл следющим образом: в $G$ выбираем подграф $G^{\circ}=\left(V, E^{\circ}\right)$, порожсенный дугами, которые удовлетворяют условию (3), и в $G^{\circ}$ фиксируем произволвное дерево с корневой верииной $v_{0}$.

Доказателъство. Согласно теореме 3 в $G$ существет дерево оптимальных путей $H^{*}=\left(V, E^{*}\right)$ с корневой вершиной $v_{0}$. Это дерево определяет оптимальную стратегию $s_{1}^{*}, s_{2}^{*}, \ldots, s_{p}^{*}$, порожденную переходами по дугам дерева $H^{*}$. Покажем, что если для каждой вершины $v \in V$ определить величины

$$
F^{i}(v)=F_{v_{0}, v}^{i}\left(s_{1}^{*}, s_{2}^{*}, \ldots, s_{p}^{*}\right),
$$

a $t(v)$ определить как число дуг пути $P_{H^{*}}\left(v_{0}, v\right)$ из $v_{0}$ в $v$, то условия (1), (2) и (3) выполняются. Докажем выполнение условия (3), поскольку выполнение условий (1) и (2) очевидно.

Пусть $V^{\circ}$ - произвольное подмножество из $\left.V, V^{\circ} \subset V\right)$, где $v_{0} \in V^{\circ}$. Если множество $V^{\circ}$ в $H^{*}$ порождает поддерево $H_{1}^{*}=\left(V^{\circ}, E_{1}^{*}\right)$ с корневой вершиной $v_{0}$, то по крайней мере для одной дуги

$$
\left(u^{\prime}, v^{\prime}\right) \in E^{*}, \quad u^{\prime} \in V^{\circ}, \quad v^{\prime} \in V \backslash V^{\circ},
$$

имеет место условие (3). Рассмотрим случай, когда $V^{\circ}$ не порождает в $H^{*}$ поддерево с корнем в $v_{0}$. Обозначим через $\bar{V}^{\circ}$ подмножество множества $V^{\circ}$ с наибольшим числом вершин, которое в $H^{*}$ порождает дерево $\bar{H}^{*}=\left(\bar{V}^{\circ}, \bar{E}^{*}\right)$ с корнем $v_{0} \in V$ (в частности, $\bar{H}^{*}$ может состоять только из вершины $v_{0}$ ). Тогда в $\bar{V}^{\circ}$ существует дуга $\left(u^{\prime}, v^{\prime}\right) \in E^{*}, u^{\prime} \in \bar{V}^{\circ}, v^{\prime} \in V \backslash \bar{V}^{\circ}$, для которой выполняется условие (3). Ясно, что $v^{\prime} \notin V^{\circ} \backslash \bar{V}^{\circ}$ потому, что $\bar{V}^{\circ}$ является наибольшим множеством в $V^{\circ}$, содержащим $v_{0}$, которое в $H^{*}$ порождает поддерево $\bar{H}^{*}=\left(\bar{V}^{\circ}, \bar{E}^{*}\right)$ с корневой вершиной $v_{0}$. Таким образом, существуют функции $F^{1}, F^{2}, \ldots, F^{p}$ и $t$ на множестве $V$, которые удовлетворяют условиям (1), (2) и (3).

Докажем теперь вторую часть теоремы. Предположим, что существуют функции $F^{1}, F^{2}, \ldots, F^{p}$ и $t$ на множестве $V$, которые обладают свойствами (1), (2) и (3). Покажем, что тогда в $G$ величины $F^{i}(v), i=1, \ldots, p$, равны значениями платежных функций

$$
F_{v_{0}, v}^{i}\left(s_{1}^{*}, s_{2}^{*}, \ldots, s_{p}^{*}\right), \quad i=1, \ldots, p,
$$

при оптимальных стратегиях $s_{1}^{*}, s_{2}^{*}, \ldots, s_{p}^{*}$ для динамической $c$-игры с начальной позицией $v_{0}$ и конечной позицией $v$. Доказательство проведем индукцией по числу вершин для динамической игровой сети. В случае $n \leqslant 2$ утверждение имеет место. Предположим, что утверждение справедливо для $n=k+1$. В $G$ выбираем вершину $v^{*}$ с наибольшим $t\left(v^{*}\right)$ и удаляем эту вершину из $G$. Полученный в результате этого граф обозначим через $G_{1}=\left(V \backslash\left\{v^{*}\right\}, E_{1}\right)$. Ясно, что в $G_{1}$ каждая вершина $v \in V \backslash\left\{v^{*}\right\}$ достижима из $v_{0}$. Легко также заметить, что для $G_{1}$ функции $F^{1}, F^{2}, \ldots, F^{p}$ и $t$ удовлетворяют условиям (1), (2) и (3), поскольку в противном случае эти функции не удовлетворяют нашим условиям в $G$. Согласно предположению 
индукции, существует дерево оптимальных путей $T_{1}^{*}=\left(V \backslash\left\{v^{*}\right\}, E_{1}^{*}\right)$ по отношению к $G_{1}$. При этом $F^{1}(v), F^{2}(v), \ldots, F^{p}(v)$ представляют собой значения платежных функций игроков в динамической $c$-игре с начальной позицией $v_{0}$ и конечной позицией $v$, при оптимальных стратегиях $s_{1}^{*}, s_{2}^{*}, \ldots, s_{p}^{*}$, то есть

$$
F^{i}(v)=F_{v_{0} v}^{i}\left(s_{1}^{*}, s_{2}^{*}, \ldots, s_{p}^{*}\right)
$$

для всех $v \in V \backslash\left\{v^{*}\right\}, i=1, \ldots, p$. Среди множества вершин $V \backslash\left\{v^{*}\right\}$ существует вершина $u^{*}$ и дуга $\left(u^{*}, v^{*}\right)$, которые удовлетворяют условию

$$
F_{v_{0} v^{*}}^{i}\left(s_{1}^{*}, s_{2}^{*}, \ldots, s_{p}^{*}\right)=F_{v_{0} u^{*}}^{i}\left(s_{1}^{*}, s_{2}^{*}, \ldots, s_{p}^{*}\right)+c_{\left(u^{*}, v^{*}\right)}^{i}\left(t\left(u^{*}\right)\right),
$$

где $t\left(u^{*}\right)$ есть число дуг в пути $P_{H^{*}}\left(v_{0}, u^{*}\right)$ из $v_{0}$ в $u^{*}$. Таким образом,

$$
F_{v_{0} v^{*}}^{i}\left(s_{1}^{*}, s_{2}^{*}, \ldots, s_{p}^{*}\right)=F^{i}\left(v^{*}\right)
$$

Теорема доказана.

\section{6. Алгоритм нахождения оптимальных стратегий в динамических $c$-играх}

Пусть задана динамическая $c$-игра, в которой граф $G$ обладает тем свойством, что любая вершина $v \in V$ достижима из $v_{0}$. Кроме того, будем считать, что в динамической игровой сети выполняется условие регулярности по отношению к каждому игроку. Тогда следующий алгоритм, который основывается на теореме 4, находит дерево $H^{*}=\left(V, E^{*}\right)$ оптимальных путей в динамической игровой сети с $p$ игроками с заданной начальной позицией $v_{0}$ и с заданными функциями стоимостей дуг $c_{e}^{i}(t)$, $e \in E, i=1, \ldots, p$. Если дерево $H^{*}$ известно, то оптимальные стратегии $s_{1}^{*}, s_{2}^{*}, \ldots, s_{p}^{*}$ игроков $1,2, \ldots, p$ могут быть найдены на основании леммы 1.

Шаг 0. Полагаем $V^{*}=\left\{v_{0}\right\}, E^{*}=\varnothing$. Каждой вершине $v \in V$ ставим в соответствие набор меток

$$
F^{1}(v), F^{2}(v), \ldots, F^{p}(v), t(v),
$$

где

$$
\begin{aligned}
F^{i}\left(v_{0}\right) & =0, \quad \forall i=1, \ldots, p \\
F^{i}(v) & =\infty, \quad \forall v \in V \backslash\left\{v_{0}\right\}, \quad i=1, \ldots, p \\
t\left(v_{0}\right) & =0, \\
t(v) & =\infty, \quad \forall v \in V \backslash\left\{v_{0}\right\} .
\end{aligned}
$$

Шаг $k$. Находим множества

$$
\begin{aligned}
E^{i} & =\left\{(\bar{u}, \bar{v}) \in E\left(V^{*}\right) \mid F^{i}(\bar{u})+c_{(\bar{u}, \bar{v})}^{i}(t(\bar{u}))\right. \\
& \left.=\min _{u \in V^{*} \cap V_{i}} \min _{v \in \bar{V}(u)}\left\{F^{i}(u)+c_{(u, v)}^{i}(t(u))\right\}\right\}, \quad i=1, \ldots, p,
\end{aligned}
$$

где

$$
\begin{aligned}
E\left(V^{*}\right) & =\left\{(u, v) \in E \mid u \in V^{*}, v \in V \backslash V^{*}\right\} \\
\bar{V}(u) & =\left\{v \in V \backslash V^{*} \mid(u, v) \in E\left(V^{*}\right)\right\}
\end{aligned}
$$


Вводим обозначение

$$
E^{\circ}=\bigcup_{i=1}^{p} E^{i}
$$

и находим множество вершин

$$
V^{\circ}=\left\{\bar{v} \in V \backslash V^{*} \mid(\bar{u}, \bar{v}) \in E^{\circ}\right\} .
$$

Затем в $V^{\circ}$ выбираем подмножество $V^{\prime}$, где $v^{\prime} \in V^{\prime}$, если в $E^{\circ}$ существует дуга $\left(u^{\prime}, v^{\prime}\right)$, для которой выполняется условие

$$
F^{i}\left(u^{\prime}\right)+c_{\left(u^{\prime}, v^{\prime}\right)}^{i}\left(t\left(u^{\prime}\right)\right)=\min _{\left(u, v^{\prime}\right) \in E^{\circ}}\left\{F^{i}(u)+c_{\left(u, v^{\prime}\right)}^{i}(t(u))\right\}, \quad i=1, \ldots, p .
$$

Для каждой вершины $v^{\prime} \in V^{\prime}$ выбираем одну дугу $\left(u^{\prime}, v^{\prime}\right) \in E^{\circ}$, которая удовлетворяет этому условию, и образуем множество $E^{\prime}$ из таких дуг. После этого находим метки $F^{i}\left(v^{\prime}\right), i=1, \ldots, p$, для вершин $v^{\prime} \in V^{\prime}$ по формуле

$$
F^{i}\left(v^{\prime}\right)=F^{i}\left(u^{\prime}\right)+c_{\left(u^{\prime}, v^{\prime}\right)}^{i}\left(t\left(u^{\prime}\right)\right), \quad\left(u^{\prime}, v^{\prime}\right) \in E^{\prime} .
$$

Значение $t\left(v^{\prime}\right)$ находим по формуле

$$
t\left(v^{\prime}\right)=t\left(u^{\prime}\right)+1, \quad\left(u^{\prime}, v^{\prime}\right) \in E^{\prime} .
$$

Заменяем множество $V^{*}$ на $V^{*} \cup V^{\prime}$, а $E^{*}$ на $E^{*} \cup E^{\prime}$. Если $V^{*} \neq V$, то переходим к шагу $k+1$, в противном случае работа алгоритма заканчивается.

Заметим, что если для заданных $e \in E, t \in\{0,1,2, \ldots, n-1\}$ и $i \in\{1,2, \ldots, p\}$ значения $c_{e}^{i}(t)$ могут быть вычислены за время $k$, то алгоритм находит дерево оптимальных путей $T^{*}=\left(V, E^{*}\right)$ за время $O\left(p n^{2} k\right)$. Действительно, каждый шаг алгоритма требует $O(p n k)$ операций и наибольшее количество шагов алгоритма равняется $n-1$. Таким образом, вычислительная сложность алгоритма в элементарных операциях есть $O\left(p n^{2} k\right)$.

\section{7. Обобщения}

В рассмотренной задаче мы предполагали, что время перехода $\tau_{u, v}$ системы $L$ из состояния $u \in V$ в $v \in V$ для всех $(u, v) \in E$ равняется единице. Легко заметить, что все результаты работы могут быть перенесены на случай, когда различным дугам $(u, v)$ и $\left(u^{\prime}, v^{\prime}\right)$ соответствуют различные длительности времени переходов $\tau_{u, v}, \tau_{u^{\prime}, v^{\prime}}$ и каждая из них может быть отлична от единицы. Теоремы 1-4 для задачи в таком общем случае также имеют место и доказываются аналогично. Поэтому, если в алгоритме заменим соотношение (5) на соотношение

$$
t\left(v^{\prime}\right)=t\left(u^{\prime}\right)+\tau_{u^{\prime} v^{\prime}}
$$

то получим алгоритм решения задачи в общем случае. Вычислительная сложность алгоритма при этом не меняется.

Более общая математическая модель динамической $c$-игры получается, если позиции игроков меняются во времени, то есть в каждый момент времени $t=0,1,2, \ldots$ задано разбиение

$$
V=V_{1}(t) \cup V_{2}(t) \cup \cdots \cup V_{p}(t),
$$

где $V_{i}(t) \cap V_{j}(t)=\varnothing, i \neq j$. Используя динамическую декомпозицию игровой сети из $[5,7]$, эту задачу можно свести к задаче из раздела 3. 


\section{Список литературы}

1. Романовский И. В., Оптимизация стационарного управления дискретным д́етерминированным процессом. Кибернетика (1967), №2, 66-78.

2. Беллман Р., Калаба Р., Динамическое программирование и современная теория управления. ИЛ, Москва, 1966.

3. Гурвич В.А., Карзанов А.В., Хачиян Л.Г., Циклические игры и нахождение минимаксных средних циклов в ориентированных графах. Журнал вычисл. матем. и матем. физики (1988) 28, №9, 1407-1417.

4. Кристофидес Н., Теория графов: алгоритмический подход. Мир, Москва, 1978.

5. Лозовану Д. Д., Алгоритмы решения некоторых классов сетевых минимаксных задач и их приложения. Кибернетика (1991), №1, 70-75.

6. Лозовану Д. Д., Трубин В. А., Задача о минимаксном пути в сети и алгоритм ее решения. Дискретная математика (1994) 6, №2, 138-144.

7. Лозовану Д. Д., Сильнополиномиальные алгоритмы поиска минимаксных путей в сетях и решение циклических игр. Кибернетика и системный анализ (1993), №5, 145-151.

8. Nash J. F., Non cooperative games. Ann. Math. (1951) 2, 286-295.

9. Мулен Э., Теория игр с примерами из математической экономики. Мир, Москва, 1985.

10. Lozovanu D., Dynamic games with $p$ players on networks. Изв. АН Республики Молдова, математика (2000) 32, №1, 41-54.

11. Boliac R., Lozovanu D., Solomon D., Optimal paths in network games with $p$ players. Discrete Appl. Math. (2000) 99, №1-3, 339-348. 